\title{
Téoros
}

Revue de recherche en tourisme

\section{La villégiature privée sur les terres publiques au Québec : un demi-siècle de mesures législatives et administratives}

\section{Danièle Laflamme}

Volume 7, numéro 2, juillet 1988

Villégiature et tourisme

URI : https://id.erudit.org/iderudit/1080413ar

DOI : https://doi.org/10.7202/1080413ar

Aller au sommaire du numéro

Éditeur(s)

Université du Québec à Montréal

ISSN

0712-8657 (imprimé)

1923-2705 (numérique)

Découvrir la revue

Citer cet article

Laflamme, D. (1988). La villégiature privée sur les terres publiques au Québec : un demi-siècle de mesures législatives et administratives. Téoros, 7(2), 27-29.

https://doi.org/10.7202/1080413ar d'utilisation que vous pouvez consulter en ligne. 


\section{La villégiature privée sur les terres publiques au Québec:}

\author{
Danièle Laflamme*
}

\section{Un demi-siècle de mesures législatives et administratives}

\section{Introduction}

L'attribution de terres de la Couronne à des particuliers pour l'établissement d'une résidence secondaire est une pratique qui a débuté, semble-t-il, dans l'entre-deuxguerres mais on ne peut retracer ses débuts avec certitude. Traditionnellement, les autorités procédaient au cas par cas: toute personne intéressée acheminait une demande à son député, à un ministre ou à une personne d'influence; l'attribution du terrain se faisait par un Arrêté en Conseil pour identifier le lot et fixer le prix de la transaction. Comme la décision d'autoriser ou non une vente était laissée à l'entière discrétion de l'autorité compétente, on peut soupçonner que des facteurs d'ordre politique jouaient un rôle prépondérant.

C'est en 1938 que l'État, face à la croissance rapide de la demande, commence à systématiser ses interventions dans ce secteur d'activités foncières. Le Conseil Exécutif ${ }^{(1)}$ délègue alors au ministère des Terres et Forêts l'autorité de délivrer des baux aux requérants de petits lots pour des fins sportives, touristiques, résidentielles et industrielles. À partir de ce moment, une série d'Arrêtés en Conseil, d'énoncés de politique et de documents gouvernementaux permettent de retracer l'évolution des différentes conditions régissant la villégiature sur les terres publiques.

\section{Le laisser-aller}

a) Mode de concession des terres

Bien qu'on manque de renseignements précis sur l'évolution de la demande à partir de 1938, on peut affirmer qu'à la fin des années. 1940 , la villégiature sur les terres publiques connaît un essor rapide.

".... le Ministère des terres et forêts a fait subdiviser, depuis une dizaine d'années, un grand nombre d'emplacements de villégiature en bordure des lacs... " (2)

Les locataires ont généralement érigé une construction sur leur lot et ont donc engagé des sommes importantes; plusieurs d'entre eux désirent devenir propriétaires. C'est pourquoi le Conseil Exécutif adopte, de 1958 a 1960 , une série de quatre décrets autorisant, pour une période limitée (un an dans chaque cas), la vente d'un nombre déterminé d'emplacements de villégiature dans certains comtés.

En 1960, on a déjà vendu tous les emplacements autorisés dans certains comtés. Comme la demande ne faiblit pas, l'autorisation de vendre des lots pour des fins de villégiature est prolongée de 5 ans. Cette fois$\mathrm{ci}$, les restrictions sautent quant au nombre d'emplacements que le Ministère peut vendre et l'autorisation est étendue "tà tous les comtés de la province où il se fait de la villégiature sur les terres de la Couronne" (3). Cette autorisation sera renouvelée en $1965^{(4)}$ aux mêmes conditions et deviendra permanente en $1970^{5}$.

Les locations, quant à elles, continuent d'obéir aux critères de 1938. Les responsables régionaux autorisent le début d'une nouvelle colonie lorsqu'ils ont reçu au moins cinq ou six demandes pour un endroit donné. Ces demandes peuvent porter sur n'importe quel plan d'eau; le choix des sites n'est pas planifié par le ministère des Terres et Forêts.

\section{b) Modalités de vente et de location} Les ventes visées par les décrets de 1958-1960 sont limitées à 5 acres et le prix minimum est de $250 \$$ l'acre $(200 \$$ l'acre dans certaines régions plus éloignées). Avant qu'un titre de propriété ne soit décerné, on doit constater sur le terrain des améliorations (dont la forme exacte ne semble pas définie) garantissant la permanence de l'établissement. II semble néanmoins que ces conditions soient plus ou moins respectées et que le ministère des Terres et Forêts n'ait pas eu, pendant longtemps, les ressources nécessaires pour exercer sur les occupants un contrôle adequat.
Notons qu'en 1965, certaines autres conditions préalables à la vente apparaissent au decret: 1) aucune vente ne peut ètre consentie sans location préalable de deux ans; 2) tout terrain, pour être vendu, doit faire partie d'une colonie estivale stable, c'est-à-dire comprennent " $\ldots$ au moins de dix $(. .$.$) a$ douze (...) chalets ou terrains loués ou patentés ${ }^{*(6)}$.

En 1970, une nouvelle condition stipule que la vente sera annulée "si, dans les cinq (...) ans suivant la vente, les constructions principales sont enlevées et non remplacées" ${ }^{*}(7)$; c'est un moyen supplémentaire de veiller au caractère permanent de l'ctablissement et de décourager la spéculation foncière. La même année, le prix de vente est majoré substantiellement (de $250 \$$ à $500 \$$ l'acre).

Les conditions de location, de leur côté, restent relativement stables jusqu'en 1970 . Depuis 1938, les baux sont d'une durée maximale de 10 ans (ils peuvent contenir une clause de renouvellement) et la superficie des lots ne doit pas dépasser 5 acres. En 1970. le loyer annuel grimpe de $15 \$$ à $35 \$$ l'acre et la superficie maximale des terrains à louer qui n'ont pas déjà été́ arpentés est réduite à 3 acres.

\section{c) Les pressions sur la ressource}

Au tournant de la décennie 1970 , les décideurs publics commencent à prendre conscience des pressions exercées sur la ressource. La villégiature pose en effet les problèmes particuliers de la privatisation des rives et de l'occupation anarchique du territoire (occupations illégales); elle constitue aussi une menace pour le milieu naturel. D'où l'urgence de définir de nouvelles règles de gestion de l'utilisation des terres publiques pour ce genre d'activités. Dès 1967 , le ministère des Terres et Forêts confie plusieurs études d'aménagement à des firmes spécialisées (11); en 1974 on crée, au sein du ministère des Terres et Forêtts, un Service de l'aménagement des terres (SAT). On espère ainsi arriver à une certaine unité de décision dans un domaine où il n'existe aucune coordination à l'échelle provinciale; jusque là, les décisions étaient laissées à chacun des responsables régionaux.

C'est donc en vue de résoudre les problèmes précités dans le contexte de la "montée générale des loisirs de plein air"(B) que les experts du SAT développent et vérifient des modeles d'aménagement des plans d'eau. Ceux-ci prévoient ${ }^{4}$ des zones de protection et des zones de développement dont une par- 
tie seulement est consacrée à la villégiature domiciliaire, le reste étant affecté à une utilisation collective" ${ }^{\text {(9) }}$.

En 1975-1976, un moratoire est imposé sur les mises en chantier de projets de villégiature domiciliaire. Il prendra fin en 1977 lorsque le nouveau gouvernement québécois présentera sa politique d'accessibilité aux terres publiques.

\section{La politique d'accessibilité de 1977}

La politique d'accessibilité aux terres publiques affirme le principe selon lequel:

${ }^{4}{ }^{4 . .}$. le sol québecois est une richesse collective qui doit être gérée pour le bénéfice de la collectivité québécoise et que conséquemment la collectivité doit y avoir accès. "Wos

$L$ attribution des lots se fait desormais par tirage au sort. La personne interessee ne peut pas concourir pour les seuls lots qui l'interessent, ce qui réduit ses chances d'obtenir celui qu'elle convoite le plus. Linsatisfaction manifestée par une bonne partie de la clientele forcera les autorités à rectifier le tir, et c'est ainsi qu'en 1979 les responsables régionaux seront de nouveau autorises à choisir le mode d'attribution ${ }^{\prime \prime \prime}$; plusieurs abandonneront le tirage au sort pour revenir à la methode du "premier arrive, premier servi".

La conséquence principale de la politique $d$ 'accessibilité pour le secteur qui nous concerme est la suppression du droit d'achat des terrains de villegiature. Ceux-ci, d'une superficie maximale de 2,5 acres, peuvent faire l'objet d'un bail à long terme (de 10 a 40 ans, renouvelable) pour un loyer annuel d'au moins $0,0165 \$ / \mathrm{m}^{2}$. Des constructions d'au moins $2000 \$$ ( $3000 \$$ dans certaines régions, dont Montréal) doivent y être érigées dans les deux premières annees du bail. Le locataire doit en plas procéder à des ameliorations foncières (deboisement, chemin, etc.). Le prix des loyers sera révise tous les cinq ans en fonction de l'indice des prix à la consommation. Ces lots sont aussi offerts par bail à court terme ( $I$ à 8 ans, renouvelable, au loyer minimum de $0.22 \$ / \mathrm{m}^{2}$ ) ou par permis d'occupation ( 12 mois ou moins). Le loyer est ajuste tous les quatre ans en fonction de l'indice des prix à la consommation.

Le principe d'accès amène aussi l'abolition des clubs prives de chasse et de pêche (1978). Ceux-ci contrólaient et développaient de grands territoires attrayants et constituaient une barrière à l'utilisation récreative du domaine public par l'ensemble de la population. Les detenteurs de baux des anciens clubs conservent un "droit interimaire collectif d'utilisation' "(liz) jusqu'à ce que le ministère des Terres et Forêts, après étude de leur dossier, leur consente un nouveau bail.

L" "opération déclubage" est consideree comme une priorite et on vise à régulariser le statut de 12000 occupants sur une période d'au plus 36 mois $^{(13)}$. Les premiers baux en règle sont émis en avril 1979.

\section{Vers la planification} des usagés du territoire

a) Les plans d'affectation

Avec la Loi sur l'aménagement et l'urbanisme (1980), l'État s'engage dans la planification globale de l'usage des terres publiques. Cet exercice débouche sur des plans d'affectation réunissant tous les usages du territoire prévus par différents ministères. Le ministère de l'Énergie et des Ressources (qui remplace en 1979 le ministère des Terres et Forêts) doit pour sa part identifier les zones propices aux activités de villégiature. Ces plans d'affectation sont soumis aux différentes Municipalités régionales de Comté et servent à la préparation des schémas d'aménagement régionaux.

Une fois les plans d'affectation établis, il faudra penser à régler le cas des occupants sans titre. Il est désormais inadmissible que des gens s'installent de façon alćatoire sur un territoire dont les différentes zones ont une vocation spécifique (forestière, récréotouristique, etc.); une telle pratique est contraire non seulement au principe de l'accès équitable sur lequel se fonde la politique d'accessibilité, mais aussi aux normes d'aménagement mises de l'avant par le ministère de l'Énergie et des Ressources. Dès 1980-1981, les fonctionnaires pressent les décideurs publics "d'amorcer la mise en oeuvre d'une solution pratique, durable et réaliste à ce vieux problème" "(14).

Le phénomène n'est d'ailleurs pas nouveau puisque le Ministère avait déjà entrepris une opération de dépistage et de poursuite des "squatters" au début des années 1970; cette démarche avait été suspendue lors de la création du SAT, le temps de préciser les objectifs du Ministère en matière de villégiature. En 1980, on estimait à 10000 leur nombre même en comptant seulement les occupations exercées par l'habitat de villegiature. Le phénomène se retrouve dans toutes les régions à des degrés variables.

\section{b) Définition de nouveaux modes d'occupation}

La villégiature résidentielle a pu croître, durant les décennies 1960 et 1970 , à cause de l'augmentation de la durée des vacances et l'clévation du niveau de vie. Mais l'essor considérable des activités de plein air, surtout dans les annees 1970 , attire vers les sites naturels une nouvelle clientèle qui désire pratiquer ces activités en dehors des grands centres. Cette catégorie d'utilisateurs fait des pressions croissantes pour l'accès aux terres publiques pour des séjours morcelés, de courte durée et ne nécessitant pas nécessairement des abris offrant tout le confort d'une résidence permanente ${ }^{(15)}$. C'est ce qui amène le ministère des Terres et Forêts à élaborer deux modes d'occupation du territoire: la villegiature concentrée et la villégiature dispersée.

\section{La villégiature concentrée}

L'expression "villégiature concentrée" désigne un développement résidentiel planifié sur le pourtour d'un lac ou le long d'un cours d'eau. Ces colonies sont généralement situées a proximite des centres, sont assez faciles d'accès et peuvent béneficier de certains services publics (eau, électricité, etc. $)^{(16)}$,

L'aménagement des plans d'eau servant à la villégiature concentrée favorise, en plus des implantations permanentes, les utilisations $\mathrm{d}^{4}$ une clientèle occasionnelle et la préservation de l'habitat naturel. Chaque projet prévoit done:

- une partie pour une colonie de villégiature:

- une partie pour des fins communautaires (récréatives);

- une partie laissée à l'état naturel.

La partie affectée à la colonie peut prendre la forme traditionnelle d'un cordon de chalets ou la forme plus récente des grappes de chalets. Les objectifs de préservation du milieu naturel amènent à imposer une bande riveraine d'au moins 30 mètres qui doit rester boisée et n'être altérée en aucune façon.

Derriere cette bande se trouve le lot en location, dont on ne peut déboiser que le tiers tout en laissant intacte une bande de 10 mètres à l'intérieur du terrain. Ces aménagements en profondeur permettent l'accès à l'eau par un sentier et l'accès à la route de desserte par un chemin de 5 mètres au maximum.

Le villégiateur doit aussi respecter les normes du ministère de l'Environnement régissant l'évacuation des eaux usées (fosses septiques) et l'installation d'un quai ou d'un débarcadère, le cas échéant.

\section{La villégiature dispersee}

La formule de la villégiature dispersée veut répondre à des besoins variés et à une demande tres forte pour une villegiature plus. rustique. Elle se pratique sur des sites plus isoles et moins accessibles par les moyens. de transport ordinaires. Les terrains ont la méme superficie que les lots de villégiature concentrée et sont soumis aux mêmes règles d'aménagement (bande riveraine, déboisement, etc.). Ils sont toutefois dépourvus de services publies et on n'y établira qu'un piedà-terre (chalet, abri ou tente-roulotte, sans fondation permanente). Il n'y a, en fait, aucune obligation de construire dans ces zones à densité d'occupation très faible.

\section{Assouplissement des normes d'accès}

a) Le rétablissement du droit d'achat

La nouvelle poltique de villégiature sur les terres publiques, adoptée en juin 1982, met en vigueur les nouvelles exigences relatives à $1^{+}$aménagement général des sites de villegiature (concentree et dispersée) et des lots eux-mèmes. Tout en réaffirmant que "l'État doit privilégier l'accessibilité au domaine public" $^{\prime \prime(17)}$, elle rétablit le droit d'achat pour les lots de villégiature concentrée (mais non pour ceux de villégiature dispersée). 
Depuis plusieurs années, on assiste en effet à une chute marquée de la demande pour les lots de villégiature concentrée. Le décret de 1977 , on l'a vu, à enlevế au locataire la possibilité d'acheter le lot qu'il occupe et sur lequel il a pourtant l'obligation de bâtir un chalet. Étant donné les coŭts de construction et le délai assez court ( 2 ans) accordé par le Ministère, plusieurs familles sont forcées de contracter une hypothèque. Or, les banques n'acceptent généralement pas (sauf un petit nombre de caisses populaires locales) de consentir un prèt hypothécaire à un particulier désirant construire sur un terrain qui ne lui appartient pas. Reste donc la formule du prêt personnel qui porte un taux d'intérêt plus élevé et qu'on n'accorde pas aussi facilement. Et même lorsque le financement ne pose pas de problème, ill n'en reste pas moins que le fait d'être privé du privilège de la propriété n'est pas de nature à sécuriser l'occupant qui a investi dans une maison et des travaux d'aménagement.

Par ailleurs, la valeur minimale des constructions à ériger dans les deux premières années du bail est portée à $6000 \$$, et la valeur minimale des améliorations foncières à $500 \$$. Autre nouveauté: comme le gouvernement juge " juste et équitable que l'Etat exige une compensation financière raisonnable pour toute concession des terres publiques ${ }^{*(18)}$ afin de couvrir les frais administratifs encourus et de procurer des revenus au Trésor public, les prix (location et vente) sont dorénavant fixés d'après l'évolution du marché foncier local, c' est-à-dire à partir de la valeur marchande des terrains.

Idéalement, cette valeur devrait être calculée pour chacun des terrains. En réalité, chaque responsable régional fixe un prix de vente général pour sa région, soit la moyenne des prix pratiqués dans les différentes zones qui la composent. Quant au loyer annuel, il est fixé à $8 \%$ de cette valeur ou au minimum prévu par l'Arrêté en Conseil dans les régions où il est impossible d'établir une valeur marchande; les prix sont ajustés annuellement selon l'indice moyen des prix a la consommation. La valeur marchande de base est révisće tous les quatre ans dans le cas des baux à court terme et tous les cinq ans pour les baux à long terme.

\section{b) Une plus grande marge de manoeuvre pour les régions}

Une fois les plans d'affectation complétés (1982), les responsables régionaux du ministère de l'Énergie et des Ressources se voient restituer le pouvoir de planifier eux-mêmes le développement des projets de villégiature: ils peuvent ainsi mieux répondre aux besoins particuliers de leur région tout en restant soumis aux normes d'aménagement et aux plans d'affectation.

Là où la demande est légère et les ressources plus abondantes, le responsable régional peut attendre que se manifeste un intérêt pour un plan d'eau pour l'ouvrir aux villégiateurs. Par contre, là où les ressources diminuent et sont d'accés plus difficile (comme c'est le cas pour la région de Montréal), on peut choisir de conditionner la demande: le responsable régional détermine d'abord quel lac sera aménagé et l'offre ensuite aux citoyens intéressés. Les responsables orientent ou non le choix selon ce qu'ils jugent être préférable pour le développement de la région et pour le futur villégiateur.

\section{c) Les occupants sans titre}

Toujours dans le cadre de sa politique de 1982 , le gouvernement adopte un autre décret ${ }^{(19)}$ visant à normaliser le statut des occupants sans titre. Tout occupant (ayant bâti sur le tertain) qui s'est fait connaître avant le ler novembre 1982 s'est vu accorder une autorisation provisoire d'une durée maximale de trois ans, à condition de payer le loyer exigé pour les baux de courte durée (ou les permis d'occupation); après étude du dossier, le Ministère a délivié un bail de courte durée après avoir constaté que l'occupation n'est pas nuisible à l'exploitation d'une ressource ou à l'exercice d'un autre droit. L'occupant ne peut toutefois pas acheter le terrain qu'il occupe. L'ampleur de la réponse des occupants sans titre a surpris tout le monde. Dans la région administrative de l'Abitibi, par exemple, alors qu'on évaluait à 1500 les cas d'occupation illegale, c'est près de 15000 demandes de régularisation qui parviennent au Ministère.

Mais malgré les efforts du Ministère pour renseigner la population, les occupations illégales se poursuivent. Un autre décret doit être adopté en $1983^{(20)}$ qui prolonge jusqu'au 31 mai 1983 la période dont disposent les "squatters"' pour déclarer leur présence. Ce délai vaut aussi pour les occupants sans titre établis depuis le 2 juin 1982, mais ceux-ci doivent en plus démontrer que leur installation est conforme aux normes du ministère de l'Énergie et des Ressources, acquitter les arrérages de loyer et payer des frais administratifs de $200 \$$. Quant aux occupants qui déclarent leur présence après le 31 mai 1983, ils devront non seulement payer des frais (400\$) et les arrérages de loyer, mais aussi prouver qu' ils étaient établis avant cette date sinon ils seront expulsés. La nouvelle législation marque la détermination du gouvernement d'enrayer cette pratique sur les terres publiques. Toutefois, comme on ne connait pas le nombre exact des occupations illégales il est difficile d'évaluer l'efficacité de ces mesures législatives.

Le rétablissement du droit d'achat n'arrive toutefois pas à redresser la demande, car la majeure partie des obstacles sont demeurés: éloignement des grands centres, hausse des loyers, hausse des coûts de construction, éloignement de la rive, etc. Si on pense aussi que cette politique est entrée en vigueur lors d'une récession économique qui gonflait les taux d'intérêt (hypothécaires ou autres), on comprend mieux pourquoi la demande a continué de chuter jusqu'en 1985. Le secteur de la villégiature dispersée, en revanche, connait une popularité croissante.

\section{Conclusion}

Ce demi-siècle de villégiature privée sur les terres publiques est un reflet intéressant de plusieurs évolutions plus ou moins parallèles: celle de la fonction publique qui, à partir de l'entre-deux-guerres et surtout de la révolution tranquille, prend de l'importance et cherche à rationaliser ses interventions; l'orientation de plus en plus socialisante des gouvernements provinciaux qui culmine avec l'arrivée au pouvoir du Parti Québécois en 1976, l'épuisement progressif d'une ressource forcément limitée (les lots de villegiature riveraine, et le milieu naturel en général), l'élévation du niveau de vie de la population, qui semble toutefois plafonner vers la fin des années 1970 et enfin, la mutation des habitudes de loisirs, qui se traduit notamment par l'intérêt pour le plein air, donc pour une villégiature plus "sauvage".

L'évolution actuelle de la situation est difficile à dépeindre faute de chiffres suffisants; mais il est certain que les facteurs précités constituent un élément d'évaluation, voire de prospective, pour les chercheurs et les décideurs dans ce domaine.

\section{Notes explicatives \\ (1) Arrete en Conseil 1379, le 9 aoūt 1938. \\ (2) Arráté en Conseil 673, le 19 juin 1958 , \\ (3) Arrété en Conseil 1361, te 30 août 1960. \\ (4) Arreaté en Conseil 2136, le 27 octobre 1965. \\ (5) Arrêté en Conseil 2985, le 5 août 1970. \\ (6) Ministère de l'Énergie et des Ressources, Colonie estivale, janvier 1977, miméo, p. 1. \\ (7) Arrêté en Consell 2985, le 5 août 1970. \\ (8) Ministère des Terres et Foráts, Rapport annuel 1976. p. 225 \\ (9) Ibidem \\ (10) Ministère de l'Ênergie et des Ressources, La villé giature sur les terres publiques. juin 1990, miméo. pp, 2-3. \\ (11) Arrêtể en Conseil 2534, le 5 septembre 1979. \\ (12) Ministere des Torres et Fonets, Rapport annuel 1977-1978, p. 133. \\ (13) Arroted en Conseil 725 , le 8 mars 1978 . \\ (14) Ministère de l'Énergie et des Ressources, Mémoire concernant les occupations sans titre, lettre de prisentation de J.-N. Poulin (sous-ministre) a $Y$. Duhaime (ministre).}

(15) Ministere de l'Énergie êt des Ressources, La villa giature sur les terres publiques, juin 1980, mimeo. p. 12 .

(16) Ministère de I'Énergie et des Ressources, La villegiature sur les terres publiques, juin 1983, brochure et dépliant.

(17) Arrèté en Conseil 1314, le 2 juin 1982

(18) Ibidem.

(19) Arrâté en Conseil 1315, le 2 juin 1982.

(20) Arrâté en Conseil 1344, le 22 juin 1983. 